\title{
FLORISTIC COMPOSITION OF OAK FORESTS AND ORIENTAL HORNBEAM SCRUBS ON MT. VIDLIČ IN THE FIRST THREE YEARS AFTER A WILDFIRE
}

\author{
Ljubinko RAKONJAC ${ }^{l}$, Marija MARKOVIĆ ${ }^{2}$, Biljana NIKOLIĆ ${ }^{1}$, \\ Aleksandar LUĆIĆ $C^{l}$, Tatjana RATKNIĆ ${ }^{l}$
}

\begin{abstract}
After a catastrophic wildfire on Vidlič Mountain in 2007, which among other things burned oak forests and hornbeam scrubs, their floristic composition was monitored for three years. Phytosociological surveys were carried out in the field using the Braun-Blanquet method, and the results are presented in the form of a synthetic phytosociological table. It was observed that in the stands in which the vegetation was only partially damaged, a shorter recovery time was required to return to the previous state - as it was before the wildfire. The initial stages of vegetation recovery at sites where complete destruction of vegetation occurred were characterized by a strong presence of annual therophytes: Orlaya grandiflora, Sideritis montana, Geranium dissectum, Althaea hirstuta. The first year after the wildfire was characterized by the dominance of annual species of Bupleurum praealtum, Crepis setosa and Centaurea calcitrap, which were not recorded in the monitored stands in the second and third year after the wildfire. In the second and third year after the wildfire, perennial plants and various grass species assumed dominance. It is necessary to conduct further systematic and continuous monitoring of the floristic composition and structure of the oak forests that completely burned in the wildfire, as well as an analysis of the profitability of the restitution of oak forests damaged by forest fires in Serbia.
\end{abstract}

Keywords: wildfire, oak forests, Oriental hornbeam scrub, Vidlič Mountain, vegetation recovery 


\title{
FIORISTIČKI SASTAV HRASTOVIH ŠUMA I ŠIBLJAKA GRABIĆA PRVE TRI GODINE NAKON POŽARA NA PLANINI VIDLIČ
}

\author{
Ljubinko RAKONJACl, Marija MARKOVIĆ ${ }^{2}$, Biljana NIKOLIĆl, \\ Aleksandar LUČIĆl, Tatjana RATKNIĆ ${ }^{l}$
}

Izvod: Nakon katastrofalnog požara na planini Vidlič 2007 godine, u kome su između ostalog izgorele hrastove šume i šibljak grabića, praćen je floristički sastav prve tri godine nakon požara. Fitocenološka istraživanja obavljena su na terenu metodom BraunBlanquet-a, a rezultati predstavljeni u vidu sintetske fitocenološke tabele.U sastojinama u kojima je došlo samo do delimičnog oštećenja vegetacije, zabeleženo je da je potrebno kraće vreme za oporavak $i$ da brže dolazi do uspostavlja prvobitnog stanja, kakvo je bilo pre požara. Početni stadijumi oporavka vegetacije na lokalitetima na kojima je došlo do potpunog uništenja vegetacije karakteristični su velikim stepenom prisutnosti jednogodišnjih terofita: Orlaya grandiflora, Sideritis montana, Geranium dissectum, Althaea hirstuta. Prva godina posle požaraje karakteristična po dominaciji jednogodišnjih vrsta Bupleurum praealtum, Crepis setosa i Centaurea calcitrapa, koje druge i treće godine nisu zabeležene u praćenim sastojinama. Druge i treće godine nakon požara dolazi do dominacije višegodišnjih biljaka i različitih vrsta iz porodice trava. Neophodna su dalja sistematska i kontinuirana praćenja florističkog sastava i strukture opožarenih hrastovih šuma koje su u potpunosti izgorele u požaru, kao i analize rentabilnost rekonstrukcije hrastovih šuma, koje su oštećene šumskim požarima u Srbiji. vegetacije

Ključne reči: požar, hrastove šume, šibljak grabića, planina Vidlič, oporavak

\footnotetext{
${ }^{1}$ Dr Ljubinko Rakonjac, Dr Biljana Nikolić, Dr Aleksandar Lučić, Dr Tatjana Ratknić Institute of Forestry, Belgrade, Serbia

${ }^{2}$ Dr Marija Marković, University of Niš, Faculty of Science and Mathematics, Niš, Serbia

Corresponding author: Marija Marković, Višegradska 33, 18000 Niš, Serbia, tel.: +381 18533015, e-mail: marijam@pmf.ni.ac.rs
}

Acknoledgement: This research was conducted as part of a project of the Ministry of Education, Science and Technological Development of the Republic of Serbia TR 31070; OI 171025. 


\section{INTRODUCTION}

The wildfire is a process of uncontrolled burning and it is considered to be the most dangerous natural force that endangers human lives and health, material goods and the environment or natural resources (Glavaš, 2003). Much has been written about the role of wildfire in the evolution and development of society and culture (Pyne, 1997; MacGregor, 2011). At the time when the Europeans reached the central parts of the American West, its forest resources seemed to be inexhaustible. However, besides performing heavy cuttings, the settlers destroyed huge forest complexes simply by burning forests in order to obtain arable land, while they used the wood ash as the first fertilizer (Frančišković, 1963). Fire, the oldest tool in the development of culture and civilization, has today turned into an ever-increasing threat to natural resources, such as forests, soil or water (Dimitrov, 1990). In $95 \%$ of cases, fires occur as a result of human action, either intentionally (up to 6\% depending from year to year) or unintentionally (about 90\%), (Šoštarić, 1989).

We are witnessing climate change that directly increases the risk of fire occurrence. The history of wildfire and the results of simulations warn us that global warming caused by wildfire can have far-reaching effects on the forests of the northern hemisphere (Dimitrov, 2000). Wildfires are behaving differently today than at any other time in human history and thus they pose a great threat to the preservation of biodiversity (TheNature Conservancy, 2004).

Wildfires can damage or in some areas completely destroy a forest ecosystem. However, forests are able to revitalize and regenerate naturally even after catastrophic fire action (Velkovski et al., 2012).

The forests on the territory of the Republic of Serbia recorded a total of 6 043 wildfires and 96239 ha of burned area in the period from 1956 to 2015. The total deforested area in the period from 1985 to 2015 amounted to 86753 hectares. Ground fires occurred on 67542 hectares and high fires on 19247 hectares. State forests accounted for $56 \%$ of the deforested area. The largest deforested area was recorded in beech stands, in the Fagetum moesiacae montanum community (40.64\%). A large burned area was also recorded in the forest of Hungarian oak and Turkey oak (Quercetum fraineto-cerris) and accounted for $29.35 \%$ of the total burned surface area (Ratknić, T., 2018).

\section{MATERIAL AND METHODS}

The thermophilic deciduous forest of Hungarian oak and Turkey oak (Quercetum frainetto-cerris Rudski 1949) is a climax community on Vidlič Mountain. However, different degradation stages of Hungarian oak and Turkey oak forests are more widely distributed on the mountain than the forests composed of densely closed stands. Marković et al. (2015) described the floristic characteristics of Oriental hornbeam scrubs - Carpinetum orientalis serbicum Rudski 1949., which grow on steep slopes and exposed rocky ridges on southern, warm and dry aspects of Vidlič Mountain. The characteristics of the vegetation growing on the burned areas of oak forests and Oriental hornbeam scrubs were monitored in the 
first (Marković et al., 2015), second (Marković et al., 2016) and third year after the wildfire (Marković et al., 2018).

Field investigations of burned thermophilic oak forests and Oriental hornbeam scrubs on Vidlič Mountain were conducted in 2008, 2009 and 2010. The result of the field research in the form of herbarized and labelled plant material was deposited in the Herbarium of the Department of Biology and Ecology of the Faculty of Natural Sciences and Mathematics, University of Niš: Herbarium Moesiacum (HMN). The collected plant material was identified according to Josifović M. (ed.) (1970-1986) and Îordanov D. (ed.) (1963-1995), and the nomenclature was harmonized with the Flora of Europe (Tutin, T. G. et al., 19641980, 1993).

Phytosociological surveys of the vegetation in the burned oak forest and Oriental hornbeam scrubs in the first, second and third year after the wildfire on Vidlič Mountain were conducted in the field using the Braun-Blanquet method (1964). The results are presented in the form of a comparative synthetic phytosociological table. The life forms of the species recorded were determined by Raunkiaer (1934). Alfa diversity of species in the community was determined using 'Flora` software package (Karadžić, B., Marinković, S., 2009). Whittaker’s species diversity was also determined: the mean value of the total number of species and the mean value of the Simpson`s Biodiversity Index (Whitaker, R.H., 1972).

\section{RESULTS AND DISCUSSION}

The oak forests and their degradation stages in the form of Oriental hornbeam scrubs on Vidlič Mountain are represented by sparse stands. The presence of ash after a wildfire increases the amount of inorganic matter, which makes the burned areas suitable for the growth of many different plants. The depth of soil is important for the growth and development of plants. The increased content of ash derived from burned plants or their parts makes the soil deeper.

There were 284 species of plants recorded on the burned areas of oak forests and Oriental hornbeam scrubs in the first, second and third year after the wildfire (Table 1). There were 139 species in the first year, 202 species in the second year, and 203 species in the third year after the wildfire.

Table 1. Comparative synthetic phytosociological table of the burned areas of oak forests and Oriental hornbeam scrubs in the first (2008), second (2009) and third (2010) year after the wildfire on Vidlič Mountain.

Legend: I-V: degree of presence, +-4: number, ch-chamaephytes, g-geophytes, hhemicryptophytes, p-phanerophytes, t-therophytes.

\begin{tabular}{|l|llll|}
\hline Species & $\begin{array}{l}1^{\text {st }} \text { year } \\
\text { after the } \\
\text { wildfire } \\
(2008)\end{array}$ & $\begin{array}{l}2^{\text {nd } y e a r} \\
\text { after the } \\
\text { wildfire } \\
(2009)\end{array}$ & $\begin{array}{l}3^{\text {rd } y e a r} \\
\text { after the } \\
\text { wildfire } \\
(2010)\end{array}$ & $\begin{array}{l}\text { Life } \\
\text { form }\end{array}$ \\
\hline $\begin{array}{l}\text { Tree layer: } \\
\text { Quercus cerris } \text { L. }\end{array}$ & $\mathrm{II}_{+-1}$ & $\mathrm{II}_{+-1}$ & $\mathrm{II}_{1}$ & $\mathrm{p}$ \\
$\begin{array}{l}\text { Quercus pubescens } \text { Willd. } \\
\text { Acer hyrcanum } \text { Fischer \& C. A. Meyer }\end{array}$ & $\mathrm{I}_{2}$ & $\mathrm{I}_{2}$ & $\mathrm{II}_{2}$ & $\mathrm{p}$ \\
$\mathrm{I}_{1}$ & $\mathrm{I}_{1}$ & $\mathrm{II}_{+-1}$ & $\mathrm{p}$
\end{tabular}


Carpinus orientalis Miller

Fraxinus ornus L.

Quercus petraea (Mattuschka) Liebl.

Rosa canina L.

Crataegus monogyna Jacq.

Crataegus laevigata (Poiret) DC. subsp.

$\mathrm{IV}+$

IV +-1

$\mathrm{I}_{+}$

laevigata

Quercus cerris L.

Acer hyrcanum Fischer \& C. A. Meyer

II +-1

$\mathrm{III}+$

$\mathrm{V}_{+-1}$

$\mathrm{II}_{+-1}$

$\mathrm{II}_{+-1}$

$\mathrm{III}+$

$\mathrm{II}_{+-1}$

II +-2

II +-2

$\mathrm{II}_{2}$

II +

II +

$\mathrm{II}_{+}$

II 1-4

$\mathrm{II}_{4}$

Malus pumila Miller

Prunus spinosa $\mathrm{L}$.

Carpinus orientalis Miller

Syringa vulgaris $\mathrm{L}$.

Chamaecytisus austriacus (L.) Link

$\mathrm{I}_{2}$

$\mathrm{I}_{2}$

II +-1

II +-2

$\mathrm{I}_{2}$

II 2

II +-4

$\mathrm{II}_{+}$

$\mathrm{I}_{+}$

II +

$\mathrm{I}_{+}$

II +

Ulmus minor Miller

Ononis pusilla L.

Ononis spinosa L.

Quercus petraea (Mattuschka) Liebl.

Fraxinus ornus L.

Viburnum lantana $\mathrm{L}$.

Clematis vitalba $\mathrm{L}$.

Sorbus torminalis (L.) Crantz

Evonymus latifolius (L.) Miller

Prunus domestica L. subsp. insititia (L.) C. K.

Schneider

Ligustrum vulgare $\mathrm{L}$.

Chamaecytisus ciliatus (Wahlenb.) Rothm.

Vinca herbacea Waldst. \& Kit.

Cytisus procumbens (Waldst. \& Kit. ex Willd.)

$\mathrm{I}_{2}$

$\mathrm{I}_{1}$

$\mathrm{I}_{3}$

$\mathrm{I}_{1}$

$\mathrm{I}_{+} \quad \mathrm{I}_{+}$

$\mathrm{I}_{+}$

$\mathrm{I}_{+}$

$\mathrm{I}_{+}$

$\mathrm{I}_{+}$

$\mathrm{I}_{+}$

$\mathrm{I}_{+}$

$\mathrm{I}_{+}$

$\mathrm{I}_{+}$

$\mathrm{I}_{+}$

$\mathrm{I}_{+}$

$\mathrm{I}_{+}$

$\mathrm{I}_{+}$

$\mathrm{I}_{+}$

$\mathrm{I}+$

$\mathrm{I}+$

$\mathrm{I}_{+}$

II +

II +

$\mathrm{I}_{+}$

$\mathrm{II}_{+}$

$\mathrm{I}_{1}$

$\mathrm{p}$

Sprengel

Ulmus procera Salisb.

Rhamnus saxatilis Jacq. subsp. tinctoria

(Waldst. \& Kit.) Nyman

Quercus pubescens Willd.

Pyrus pyraster Burgsd.

Genista sericea Wulfen

Rubus idaeus $\mathrm{L}$.

Pyrus amygdaliformis Vill.

Ground flora layer:

Orlaya grandiflora (L.) Hoffm.

Medicago sativa L. subsp. falcata (L.)

Arcangeli

Sideritis montana $\mathrm{L}$.

Geranium dissectum $\mathrm{L}$.

Althaea hirsuta L.

Euphorbia cyparissias L.

Carduus candicans Waldst. \& Kit. subsp.

candicans

Asperula purpurea (L.) Ehrend.

Dactylis glomerata L.

Brachypodium pinnatum (L.) Beauv.

Poa pratensis $\mathrm{L}$.

Fragaria vesca $\mathrm{L}$.

$I_{1}$

$\mathrm{I}_{+}$

$\mathrm{I}_{2}$

$\mathrm{I}+$

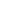

.

I +-1

$\mathrm{I}_{+}$

$\mathrm{I}_{+}$

$\mathrm{I}_{+}$

$\mathrm{I}_{+}$

ch

.

.

$\mathrm{V}_{+-2}$

IV +-2

$\mathrm{V}_{+}$

$\mathrm{V}_{+}$

$\mathrm{V}_{+-1}$

$\mathrm{h}$

$\mathrm{p}$

$\begin{array}{lll}\mathrm{IV}_{+-2} & \mathrm{IV} \\ + & \mathrm{III}_{+} & \mathrm{t}\end{array}$

$\mathrm{IV}_{+-1}$

$\mathrm{III}_{+}$

$\mathrm{IV}_{+-1}$

$\mathrm{III}_{+}$

$\mathrm{III}_{+}$

$\mathrm{III}_{+-1}$

$\mathrm{III}_{+}$

$\mathrm{II}_{+}$

III+

IV +-2

$\mathrm{V}_{+-2}$

IV +1

$\mathrm{V}_{+}$

h

$\mathrm{III}_{1-2}$

$\mathrm{IV}_{+-1}$

$\mathrm{IV}_{+-1}$

III $_{+-1}$

IV +-1

IIII-2

III $2-4$

IIII-3

III +-2

III +-2

$\mathrm{III}_{+}$

III $_{+-2}$

$\mathrm{III}_{+-2}$

$\mathrm{ch}$
$\mathrm{h}$
$\mathrm{h}$
$\mathrm{h}$ 
Teucrium chamaedrys L.

Helleborus odorus Waldst. \& Kit.

Eryngium campestre L.

Agrimonia eupatoria $\mathrm{L}$.

Coronilla varia $\mathrm{L}$.

Convolvulus cantabrica $\mathrm{L}$.

Linaria vulgaris Miller

Galium aparine $\mathrm{L}$.

Festuca valesiaca Schleicher ex Gaudin

Vincetoxicum hirundinaria Medicus

Lactuca serriola $\mathrm{L}$.

Allium scorodoprasum L. subsp. rotundum

(L.) Stearn

Acanthus balcanicus Heywood \& I. B. K.

Richardson

Carex humilis Leysser

Helianthemum nummularium (L.) Miller

Satureja kitaibelii Wierzb.

Teucrium montanum $\mathrm{L}$.

Verbascum lychnitis L.

Campanula bononiensis $\mathrm{L}$.

Melica ciliata $\mathrm{L}$.

Thlaspi perfoliatum $\mathrm{L}$.

Cuscuta europaea $\mathrm{L}$.

Cuscuta approximata Bab.

Myosotis arvensis (L.) Hill

Trifolium alpestre $\mathrm{L}$.

Astragalus onobrychis L.

Viola jordanii Hanry

Hypericum perforatum $\mathrm{L}$.

Leontodon hispidus $\mathrm{L}$.

Clinopodium vulgare $\mathrm{L}$.

Thymus pannonicus All.

Thymus glabrescens Willd.

Potentilla recta $\mathrm{L}$.

Artemisia alba Turra

Allium sphaerocephalon $\mathrm{L}$.

Digitalis lanata Ehrh.

Achillea millefolium L.

Cruciata glabra (L.) Ehrend.

Asperula cynanchica $\mathrm{L}$.

Stachys germanica L.

Lapsana communis L.

Cerastium brachypetalum Pers.

Muscari neglectum Guss. ex Ten.

Crupina vulgaris Cass.

Chrysopogon gryllus (L.) Trin.

Trifolium pratense $\mathrm{L}$.

Glechoma hirsuta Waldst. \& Kit.

Poa angustifolia $\mathrm{L}$.

Euphorbia seguierana Necker subsp. niciciana

$\mathrm{III}_{+-1}$

$\mathrm{III}_{+-1}$

III $_{+-2}$

III+-1

$\mathrm{III}_{1}$

III+-1

ch

$\mathrm{III}_{+}$

$\mathrm{III}_{+}$

$\mathrm{III}_{+}$

$\mathrm{II}_{+} \quad \mathrm{III}_{+}$

$\mathrm{III}_{+}$

$\mathrm{II}+$

$\mathrm{III}_{+}$

$\mathrm{III}_{+}$

II $1-2$

III +-2

$\mathrm{III}_{1}$

II +

$\mathrm{III}_{+}$

$\mathrm{III}_{+}$

II +-2

II +-2

IIII $_{+-1}$

II +

II +-2

$\mathrm{II}_{+}$

$\mathrm{II}_{+}$

III $_{+-2}$

$\mathrm{II}_{+}$

$\mathrm{II}_{+}$

$\mathrm{III}_{+}$

$\mathrm{II}_{+}$

II +

III $_{+}$

III+

$\mathrm{h}$

$\mathrm{II}_{+}$

II +

II +

$\mathrm{III}_{+}$

ch

II 1-2

$\mathrm{II}_{2}$

II $_{1}$

h

II +-1

II +

II 1-2

ch

II +-1

$\mathrm{II}_{1}$

II 1

ch

II +

II +

II +

ch

II +

II +-1

II +

$\mathrm{h}$

II +-1

$\mathrm{II}_{+}$

$\mathrm{II}_{+}$

II +-1

$\mathrm{III}_{+}$

$I_{1}$

II +-2

$\mathrm{II}_{+}$

I+

I+

I+

$I_{1}$

$\mathrm{I}_{+}$

$\mathrm{I}_{1}$

I +

I+

I +

$\mathrm{I}_{+}$

I+

$\mathrm{I}_{+}$

I +

$\mathrm{I}_{1}$

$I_{1}$

$\mathrm{I}_{+}$

I+

I+

I +

$\mathrm{I}_{+}$

$\mathrm{I}_{+}$

$\mathrm{I}_{2}$

$\mathrm{I}_{1}$

I +

I +

I +

I+

$\mathrm{I}_{+}$

$\mathrm{III}_{+}$

$\mathrm{I}_{+}$

$\mathrm{I}_{+}$

$\mathrm{III}_{+}$

III $_{+-1}$

$\mathrm{III}_{+-1}$

$\mathrm{II}_{+-1}$

IV +

II +-2

IIII+1 $_{+1}$

$\mathrm{II}_{+-1}$

$\mathrm{III}_{+}$

$\mathrm{II}_{+}$

$\mathrm{III}_{+}$

II +-1

II 1-2

$\mathrm{II}_{+}$

$\mathrm{II}_{+}$

$\mathrm{II}_{+}$

$\mathrm{II}_{+}$

$\mathrm{II}_{+}$

$\mathrm{II}_{+}$

$\mathrm{II}_{+-1}$

II +-3

$\mathrm{II}_{+-1}$

$\mathrm{II}_{+}$

II +

II +

II +

$\mathrm{II}_{+}$

$\mathrm{II}_{+}$

$\mathrm{I}_{2}$

$\mathrm{I}_{+}$

$\mathrm{I}_{2}$

$\mathrm{I}_{1}$

$\mathrm{I}_{1}$

$\mathrm{h}$

(Borbás ex Novák) Rech.

Geum urbanum L.

Carex caryophyllea Latourr.

Marrubium peregrinum $\mathrm{L}$.

Ornithogalum pyrenaicum $\mathrm{L}$

Allium flavum L.

$\mathrm{I}_{+}$

$\mathrm{I}_{+}$

$\mathrm{I}_{+}$

$\mathrm{I}_{+}$

$\mathrm{I}_{+}$

$\begin{array}{ll}\mathrm{I}_{+} & \mathrm{h} \\ \mathrm{I}_{+} & \mathrm{h} \\ \mathrm{I}_{+} & \mathrm{g} \\ \mathrm{I}_{+} & \mathrm{g} \\ \mathrm{I}_{+} & \mathrm{g}\end{array}$




\begin{tabular}{|c|c|c|c|c|}
\hline Viola alba Besser & $\mathrm{I}_{+}$ & $\mathrm{I}_{+}$ & $\mathrm{I}_{+}$ & $\mathrm{h}$ \\
\hline Verbascum speciosum Schrader & $\mathrm{I}_{+}$ & $\mathrm{I}_{+}$ & $\mathrm{I}_{+}$ & $\mathrm{h}$ \\
\hline Arabis recta Vill. & $\mathrm{II}+$ & $\mathrm{I}_{+}$ & . & $\mathrm{t}$ \\
\hline $\begin{array}{l}\text { Ajuga chamaepitys (L.) Schrebersubsp. chia } \\
\text { (Schreber) Arcangeli }\end{array}$ & $\mathrm{I}_{+}$ & $\mathrm{II}_{+}$ & - & $\mathrm{t}$ \\
\hline Thesium arvense Horvatovszky & $\mathrm{I}_{+}$ & $\mathrm{II}_{+}$ & - & $\mathrm{h}$ \\
\hline Carduus acanthoides L. & $\mathrm{I}_{2}$ & $\mathrm{I}_{+}$ & - & $\mathrm{h}$ \\
\hline Thalictrum aquilegifolium $\mathrm{L}$. & $\mathrm{I}_{1}$ & $\mathrm{I}_{2}$ & . & $\mathrm{h}$ \\
\hline Dichanthium ischaemum (L.) Roberty & $\mathrm{I}_{+}$ & $\mathrm{I}_{1}$ & . & $\mathrm{h}$ \\
\hline Trifolium scabrum L. & $\mathrm{I}_{+}$ & $\mathrm{I}_{+}$ & . & $\mathrm{t}$ \\
\hline Veronica austriaca L. subsp. austriaca & $\mathrm{I}_{+}$ & $\mathrm{I}_{+}$ & . & $\mathrm{ch}$ \\
\hline $\begin{array}{l}\text { Linaria genistifolia (L.) Miller subsp. sofiana } \\
\text { (Velen.) Chater \& D. A. Webb }\end{array}$ & $\mathrm{I}_{+}$ & $\mathrm{I}_{+}$ & - & $\mathrm{h}$ \\
\hline Euphorbia falcata $\mathrm{L}$. & $\mathrm{I}_{+}$ & $\mathrm{I}_{+}$ & - & $\mathrm{t}$ \\
\hline Crucianella angustifolia $\mathrm{L}$. & $\mathrm{I}_{+}$ & $\mathrm{I}_{+}$ & • & $\mathrm{t}$ \\
\hline Campanula trichocalycina Ten. & $\mathrm{I}_{+}$ & $\mathrm{I}_{+}$ & - & $\mathrm{h}$ \\
\hline Bromus commutatus Schrader & $\mathrm{I}_{+}$ & $\mathrm{I}_{+}$ & - & $\mathrm{t}$ \\
\hline Trifolium badium Schreber & $\mathrm{I}_{+}$ & $\mathrm{I}_{+}$ & - & $\mathrm{h}$ \\
\hline Torilis arvensis (Hudson) Link & $\mathrm{I}_{+}$ & $\mathrm{I}_{+}$ & . & $\mathrm{t}$ \\
\hline $\begin{array}{l}\text { Crepis foetida L. subsp.rhoeadifolia (Bieb.) } \\
\text { Čelak. }\end{array}$ & $\mathrm{II}+$ & - & $\mathrm{II}_{+-1}$ & $\mathrm{t}$ \\
\hline Fallopia convolvulus (L.) Á. Löve & $\mathrm{I}_{1}$ & . & $\mathrm{I}_{+}$ & $\mathrm{t}$ \\
\hline Torilis japonica (Houtt.) DC. & $\mathrm{I}_{1}$ & . & $\mathrm{I}_{+}$ & $\mathrm{t}$ \\
\hline Vicia lathyroides L. & $\mathrm{I}_{1}$ & . & $\mathrm{I}_{+}$ & $\mathrm{t}$ \\
\hline Sonchus asper (L.) Hill & $\mathrm{I}_{+}$ & . & $\mathrm{I}_{+}$ & $\mathrm{t}$ \\
\hline $\begin{array}{l}\text { Centaurea biebersteinii DC. subsp. australis } \\
\text { (Pančić) Dostál }\end{array}$ & $\cdot$ & $\mathrm{IV}_{+}$ & $\mathrm{IV}_{+}$ & $\mathrm{h}$ \\
\hline Medicago lupulina $\mathrm{L}$. & . & $\mathrm{III}_{+-2}$ & $\mathrm{IV}_{+}$ & $\mathrm{t}$ \\
\hline Vicia sativa L. subsp. nigra (L.) Ehrh. & . & $\mathrm{II}_{+}$ & $\mathrm{III}_{+}$ & $\mathrm{t}$ \\
\hline Agrostis capillaris $\mathrm{L}$. & . & $\mathrm{II}_{+-2}$ & $\mathrm{II}_{2}$ & $\mathrm{~h}$ \\
\hline Festuca panciciana (Hackel) K. Richter & . & $\mathrm{II}_{+-2}$ & $\mathrm{II}_{2}$ & $\mathrm{~h}$ \\
\hline Potentilla argentea $\mathrm{L}$. & . & $\mathrm{II}_{+}$ & $\mathrm{II}_{+}$ & $\mathrm{h}$ \\
\hline Medicago minima (L.) Bartal. & . & $\mathrm{II}_{+-2}$ & $\mathrm{I}_{2}$ & $\mathrm{t}$ \\
\hline Trifolium campestre Schreber & . & $\mathrm{II}+$ & $\mathrm{I}_{+}$ & $\mathrm{t}$ \\
\hline Viola hirta $\mathrm{L}$. & . & $\mathrm{II}+$ & $\mathrm{I}_{+}$ & $\mathrm{h}$ \\
\hline Viola kitaibeliana Schultes & . & $\mathrm{II}+$ & $\mathrm{I}_{+}$ & $\mathrm{t}$ \\
\hline Stachys recta $\mathrm{L}$. & . & $\mathrm{I}_{+}$ & $\mathrm{III}_{+}$ & $\mathrm{h}$ \\
\hline Fragaria viridis Duchesne & . & $\mathrm{I}_{+-3}$ & $\mathrm{II}_{+-3}$ & $\mathrm{~h}$ \\
\hline Achillea crithmifolia Waldst. \& Kit. & . & $\mathrm{I}_{+-1}$ & $\mathrm{II}+$ & $\mathrm{g}$ \\
\hline Ajuga laxmannii (L.) Bentham & . & $\mathrm{I}_{+-1}$ & $\mathrm{II}+$ & $\mathrm{g}$ \\
\hline Verbascum phlomoides L. & . & $\mathrm{I}_{1}$ & $\mathrm{II}_{+}$ & $\mathrm{h}$ \\
\hline Prunella laciniata (L.) L. & . & $\mathrm{I}_{+}$ & $\mathrm{II}_{+}$ & $\mathrm{h}$ \\
\hline Bromus squarrosus L. & . & $\mathrm{I}_{+}$ & $\mathrm{II}_{+}$ & $\mathrm{t}$ \\
\hline Hypericum rumeliacum Boiss. & . & $\mathrm{I}_{+}$ & $\mathrm{II}_{+}$ & $\mathrm{h}$ \\
\hline Ptilostemon afer (Jacq.) W. Greuter & • & $\mathrm{I}_{+}$ & $\mathrm{II}_{+}$ & $\mathrm{t}$ \\
\hline Origanum vulgare $\mathrm{L}$. & . & $\mathrm{I}_{+}$ & $\mathrm{II}_{+-1}$ & $\mathrm{~g}$ \\
\hline Muscari comosum (L.) Miller & . & $\mathrm{I}_{+}$ & $\mathrm{II}_{+}$ & $\mathrm{g}$ \\
\hline Galium mollugo L. & . & $\mathrm{I}_{+}$ & $\mathrm{II}_{+}$ & $\mathrm{h}$ \\
\hline $\begin{array}{l}\text { Linaria rubioides Vis. \& Pančić subsp. } \\
\text { nissana Niketić \& Tomović }\end{array}$ & . & $\mathrm{I}_{+}$ & $\mathrm{II}_{+}$ & $\mathrm{h}$ \\
\hline Onobrychis alba (Waldst. \& Kit.) Desv. & . & $\mathrm{I}_{+}$ & $\mathrm{II}_{+}$ & $\mathrm{h}$ \\
\hline Dasypyrum villosum (L.) P. Candargy & • & $\mathrm{I}_{1}$ & $\mathrm{I}_{1}$ & $\mathrm{t}$ \\
\hline Vicia hirsuta (L.) S. F. Gray & - & $\mathrm{I}_{1}$ & $\mathrm{I}_{+}$ & $\mathrm{t}$ \\
\hline Acinos alpinus (L.) Moench subsp. & - & $\mathrm{I}_{+}$ & $\mathrm{I}_{+}$ & $\mathrm{h}$ \\
\hline Majoranifolius (Miller) P. W. Ball & & & & \\
\hline Acinos arvensis (Lam.) Dandy & . & $\mathrm{I}_{+}$ & $\mathrm{I}_{+}$ & $\mathrm{t}$ \\
\hline Aethionema saxatile (L.) R. Br. & • & $\mathrm{I}_{+}$ & $\mathrm{I}_{+}$ & $\mathrm{ch}$ \\
\hline
\end{tabular}




\begin{tabular}{|c|c|c|c|c|}
\hline Arabidopsis thaliana (L.) Heynh. & . & $\mathrm{I}_{+}$ & $\mathrm{I}_{+}$ & $\mathrm{t}$ \\
\hline Carex brevicollis DC. & . & $\mathrm{I}_{+}$ & $\mathrm{I}_{+}$ & $\mathrm{h}$ \\
\hline Carlina vulgaris $\mathrm{L}$. & . & $\mathrm{I}_{+}$ & $\mathrm{I}_{+}$ & $\mathrm{h}$ \\
\hline Lactuca saligna $\mathrm{L}$. & . & $\mathrm{I}_{+}$ & $\mathrm{I}_{+}$ & $\mathrm{t}$ \\
\hline Lens nigricans (Bieb.) Godron & . & $\mathrm{I}_{+}$ & $\mathrm{I}_{+}$ & $\mathrm{t}$ \\
\hline Lotus corniculatus $\mathrm{L}$. & . & $\mathrm{I}_{+}$ & $\mathrm{I}_{+}$ & $\mathrm{h}$ \\
\hline Melica transsilvanica Schur & . & $\mathrm{I}_{+}$ & $\mathrm{I}_{+}$ & $\mathrm{h}$ \\
\hline Orobanche loricata Reichenb. & . & $\mathrm{I}_{+}$ & $\mathrm{I}_{+}$ & g \\
\hline Plantago argentea Chaix & . & $\mathrm{I}_{+}$ & $\mathrm{I}_{+}$ & $\mathrm{h}$ \\
\hline Plantago lanceolata $\mathrm{L}$. & . & $\mathrm{I}_{+}$ & $\mathrm{I}_{+}$ & $\mathrm{h}$ \\
\hline Reseda lutea $\mathrm{L}$ & . & $\mathrm{I}_{+}$ & $\mathrm{I}_{+}$ & $\mathrm{h}$ \\
\hline Rumex sanguineus $\mathrm{L}$. & . & $\mathrm{I}_{+}$ & $\mathrm{I}_{+}$ & $\mathrm{h}$ \\
\hline Salvia austriaca Jacq. & . & $\mathrm{I}_{+}$ & $\mathrm{I}_{+}$ & $\mathrm{h}$ \\
\hline Salvia nemorosa L. & . & $\mathrm{I}_{+}$ & $\mathrm{I}+$ & $\mathrm{h}$ \\
\hline Sanguisorba minor Scop. & . & $\mathrm{I}_{+}$ & $\mathrm{I}_{+}$ & $\mathrm{h}$ \\
\hline Scabiosa argentea $\mathrm{L}$. & . & $\mathrm{I}_{+}$ & $\mathrm{I}_{+}$ & $\mathrm{h}$ \\
\hline Valerianella dentata (L.) Pollich & . & $\mathrm{I}+$ & $\mathrm{I}+$ & $\mathrm{t}$ \\
\hline Ajuga genevensis L. & . & $\mathrm{I}_{+}$ & $\mathrm{I}_{+}$ & $\mathrm{h}$ \\
\hline Convolvulus arvensis $\mathrm{L}$. & . & $\mathrm{I}_{+}$ & $\mathrm{I}_{+}$ & $\mathrm{g}$ \\
\hline Cruciata laevipes Opiz & . & $\mathrm{I}_{+}$ & $\mathrm{I}_{+}$ & $\mathrm{g}$ \\
\hline Lathyrus venetus (Miller) Wohlf. & . & $\mathrm{I}_{+}$ & $\mathrm{I}_{+}$ & $\mathrm{g}$ \\
\hline Poa compressa $\mathrm{L}$. & . & $\mathrm{I}_{+}$ & $\mathrm{I}_{+}$ & $\mathrm{h}$ \\
\hline Sedum acre $\mathrm{L}$. & . & $\mathrm{I}_{+}$ & $\mathrm{I}_{+}$ & $\mathrm{g}$ \\
\hline Petrorhagia saxifraga (L.) Link & . & $\mathrm{I}_{+}$ & $\mathrm{I}_{+}$ & ch \\
\hline Bupleurum praealtum $\mathrm{L}$. & $\mathrm{IV}_{+-1}$ & . & . & $\mathrm{t}$ \\
\hline Crepis setosa Haller & $\mathrm{III}_{+}$ & . & . & $\mathrm{t}$ \\
\hline Centaurea calcitrapa $\mathrm{L}$. & II +-4 & . & . & $\mathrm{t}$ \\
\hline Festuca pratensis Hudson & II +-2 & . & . & $\mathrm{h}$ \\
\hline Ononis pusilla $\mathrm{L}$. & $\mathrm{II}+$ & . & - & ch \\
\hline Lepidium campestre (L.) R. Br. & $\mathrm{I}_{1}$ & . & . & $\mathrm{t}$ \\
\hline Bromus erectus Hudson & $\mathrm{I}_{+-1}$ & . & . & $\mathrm{h}$ \\
\hline Achillea pannonica Scheele & $\mathrm{I}_{+}$ & . & . & $\mathrm{h}$ \\
\hline Agropyron cristatum (L.) Gaertner & $\mathrm{I}_{+}$ & . & . & $\mathrm{h}$ \\
\hline Calystegia sepium (L.) R. Br. & $\mathrm{I}_{+}$ & . & . & $\mathrm{ch}$ \\
\hline Camelina rumelica Velen. & $\mathrm{I}_{+}$ & . & . & $\mathrm{t}$ \\
\hline Cirsium arvense (L.) Scop. & $\mathrm{I}_{+}$ & . & . & $\mathrm{g}$ \\
\hline Centaurium erythraea Rafn & $\mathrm{I}_{+}$ & . & . & $\mathrm{t}$ \\
\hline Coronilla scorpioides (L.) Koch & $\mathrm{I}_{+}$ & . & . & $\mathrm{t}$ \\
\hline Daucus carota $\mathrm{L}$. & $\mathrm{I}_{+}$ & . & . & $\mathrm{t}$ \\
\hline Festuca heterophylla Lam. & $\mathrm{I}_{+}$ & . & . & $\mathrm{h}$ \\
\hline Festuca ovina $\mathrm{L}$. & $\mathrm{I}_{+}$ & . & . & $\mathrm{h}$ \\
\hline Galium verum $\mathrm{L}$. & $\mathrm{I}_{+}$ & . & . & g \\
\hline Himantoglossum hircinum (L.) Sprengel & $\mathrm{I}_{+}$ & . & . & $\mathrm{g}$ \\
\hline Lathyrus venetus (Miller) Wohlf. & $\mathrm{I}_{+}$ & . & . & $\mathrm{g}$ \\
\hline Melica uniflora Retz. & $\mathrm{I}_{+}$ & . & . & g \\
\hline Nigella arvensis $\mathrm{L}$. & $\mathrm{I}_{+}$ & . & . & $\mathrm{t}$ \\
\hline Petrorhagia prolifera (L.) P. W. Ball \& & $\mathrm{I}_{+}$ & . & . & $\mathrm{t}$ \\
\hline Heywood & & & & \\
\hline Stellaria media (L.) Vill. & $\mathrm{I}_{+}$ & . & . & $\mathrm{t}$ \\
\hline Tamus communis $\mathrm{L}$. & $\mathrm{I}_{+}$ & . & . & $\mathrm{g}$ \\
\hline Vicia sativa L. subsp. sativa & $\mathrm{I}_{+}$ & . & . & $\mathrm{h}$ \\
\hline Vicia tetrasperma (L.) Schreber & $\mathrm{I}_{+}$ & . & . & $\mathrm{t}$ \\
\hline Viola odorata $\mathrm{L}$ & $\mathrm{I}_{+}$ & . & . & $\mathrm{h}$ \\
\hline Viola tricolor $\mathrm{L}$. & $\mathrm{I}_{+}$ & . & . & $\mathrm{h}$ \\
\hline $\begin{array}{l}\text { Scleranthus perennis L. subsp. dichotomus } \\
\text { (Schur) Nyman }\end{array}$ & . & $\mathrm{II}_{+-1}$ & • & $\mathrm{h}$ \\
\hline Erysimum diffusum Ehrh. & . & $\mathrm{II}_{+}$ & . & $\mathrm{h}$ \\
\hline
\end{tabular}




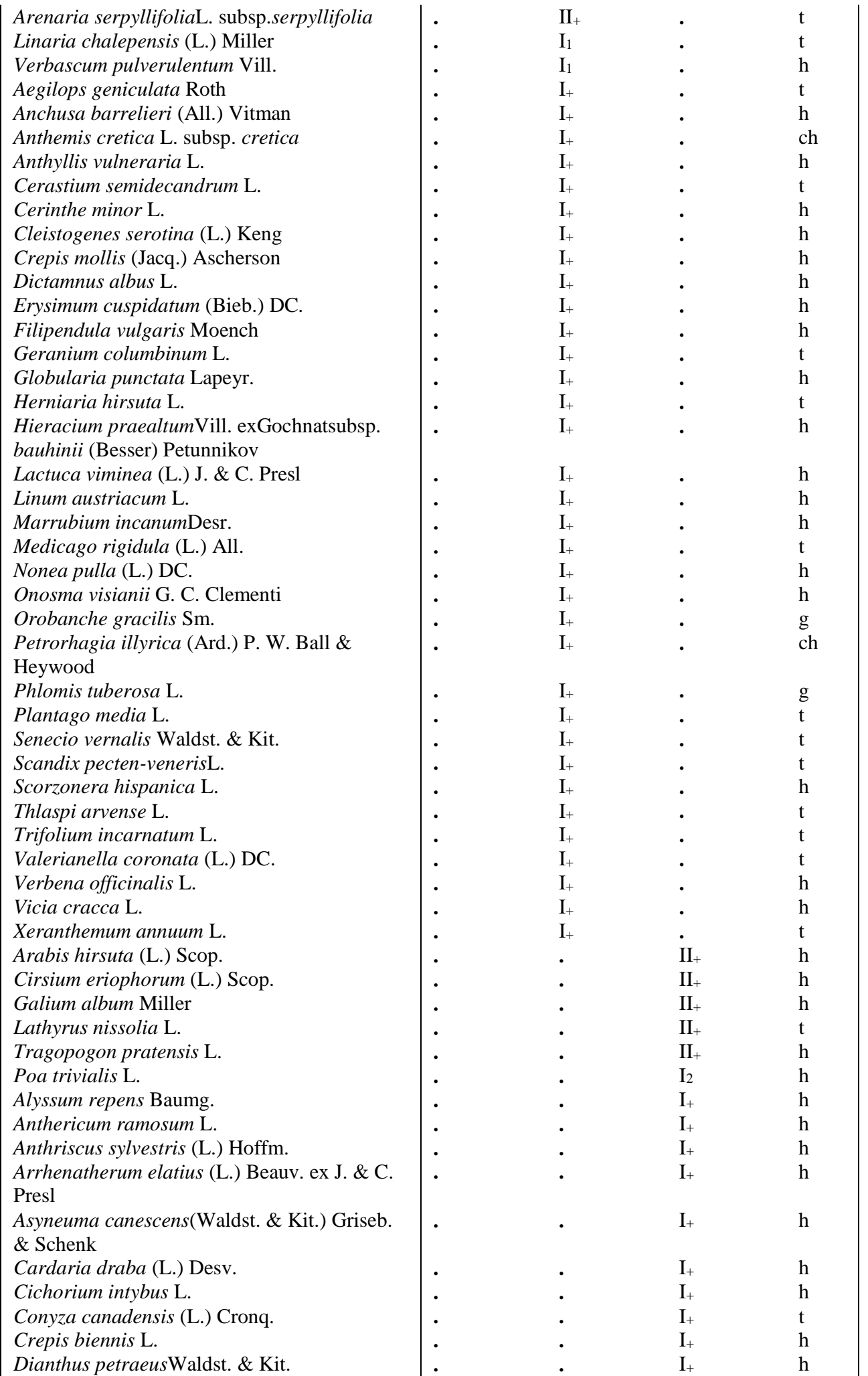


Draba muralis L.

Dorycnium pentaphyllum Scop. subsp.herbaceum(Vill.) Rouy

Epipactis helleborine (L.) Crantz

Epilobium angustifolium $\mathrm{L}$.

Erigeron acer L.

Fumaria officinalis $\mathrm{L}$.

Galeopsis ladanum L.

Geranium sanguineum $\mathrm{L}$.

Hieracium barbatum Tausch

Koeleria nitidula Velen.

Lathyru spallescens (Bieb.) C. Koch

Leontodon crispus Vill.

Logfia minima (Sm.) Dumort.

Minuartia verna (L.) Hiern

Mycelis muralis (L.) Dumort.

Ornithogalum pyramidale $\mathrm{L}$.

Peucedanum alsaticum $\mathrm{L}$.

Prunella vulgaris $\mathrm{L}$.

Ranunculus illyricus L.

Sherardia arvensis $\mathrm{L}$.

Silene noctiflora $\mathrm{L}$.

Sonchus arvensis $\mathrm{L}$.

Stipa pulcherrima C. Koch

Torilis leptophylla (L.) Reichenb.

Veronica chamaedrys L.

Vicia incana Gouan

Viola arvensis Murray

The tree layer had 7 common species in the first, second and third year after the wildfire. These were: Quercus cerris, Quercus pubescens, Acer hyrcanum, Carpinus orientalis, Fraxinus ornus, Quercus petraea and Pyrus pyraster. The shrub layer recorded 33 species, 22 of which were present in the first, second and third year after the wildfire, six species were present in the second and third year after the wildfire, and only five species were present in the third year after the wildfire.

The ground flora layer had 67 species present in all three years of monitoring. The first and the second year after the wildfire had 15 species in common. The first and the third year after the wildfire had five common species, while the second and third year after the wildfire had 53 common species. There were 29 species present only in the first year, 39 species only in the second year and 43 species only in the third year after the wildfire.

The species that were found in all three years of monitoring had the greatest share (101 species or $34.95 \%)$. They were followed by the group of plants recorded in the second and third year after the wildfire (59 species or 20.42\%). A group of plants recorded in the first and second year after the wildfire had a smaller share (15 species or 5.19\%), while the plants that were present in the first and third year after the wildfire had the smallest share (5 species or $1.73 \%$ ).

The mean value of the total number of species and the mathematical value of Simpson`s biodiversity index on the burned area of oak forests and Oriental hornbeam scrubs were growing year by year. Compared to the mean number of species on the areas not affected by the wildfire, they were smaller only in the first 
year after the wildfire, while in the second and third year after the wildfire they were higher than the mean number of species on the areas not affected by the wildfire (Table 2).

Table 2. Mean values of the total number of species and Simpson's index of biodiversity of the vegetation of oak forests and Oriental hornbeam scrubs in the first, second and third year after the wildfire on Vidlic Mountain and the comparison with the corresponding areas not affected by the wildfire

\begin{tabular}{|c|c|c|c|c|}
\hline & $\begin{array}{l}\text { Vegetation not } \\
\text { affected by the } \\
\text { wildfire }\end{array}$ & $\begin{array}{l}1^{\text {st }} \text { year after } \\
\text { the wildfire } \\
(2008)\end{array}$ & $\begin{array}{l}2^{\text {nd }} \text { year after } \\
\text { the wildfire } \\
(2009) \\
\text { (209) }\end{array}$ & $\begin{array}{l}3^{\text {rd }} \text { year after } \\
\text { the wildfire } \\
(2010)\end{array}$ \\
\hline $\begin{array}{l}\text { Mean value of } \\
\text { the } \\
\text { number } \\
\text { species }\end{array}$ & 38.75 & 38.5 & 52.8 & 53.43 \\
\hline $\begin{array}{l}\text { Mean value of } \\
\text { Simpson's } \\
\text { index of } \\
\text { biodiversity }\end{array}$ & 0.968 & 0.967 & 0.976 & 0.977 \\
\hline
\end{tabular}

Source: Marković et al., 2015; Marković et al., 2015; Marković et al., 2016;

Marković et al., 2018

An analysis of the percentage share of life forms on the burned areas of oak forests and Oriental hornbeam scrubs in the first three years after the wildfire reveals their hemicryptophytic-therophytic character (Table 3), which is a general characteristic of the flora of the majority of regions in the temperate zone (Diklić, 1984). A higher percentage of annual therophytes was recorded in the first year than in the second and third year after the wildfire. Their seeds can be brought to the burned area by various means (by wind or animals), where they find favorable conditions for their growth. The share of therophytes decreases in the second and third year, and they are replaced by other, perennial plant species.

Table 3. Overview of the percentage share of life forms on the burned areas of oak forests and Oriental hornbeam scrubs in the first, second and third year after the wildfire on Vidlič Mountain

Legend: ch-chamaephytes, g-geophytes, h-hemicryptophytes, p-phanerophytes, ttherophytes.

\begin{tabular}{|l|l|l|l|}
\hline \multicolumn{1}{|c|}{ Life form } & \multicolumn{1}{|c|}{$\begin{array}{c}1^{\text {st }} \text { year after the } \\
\text { wildfire (2008) }\end{array}$} & $\begin{array}{c}2^{\text {nd }} \text { year after the } \\
\text { wildfire (2009) }\end{array}$ & $\begin{array}{c}3^{\text {rd }} \text { year after the } \\
\text { wildfire (2010) }\end{array}$ \\
\hline $\mathrm{ch}(\%)$ & 10.71 & 8.82 & 8.87 \\
\hline $\mathrm{g}(\%)$ & 9.28 & 8.82 & 9.85 \\
\hline $\mathrm{h}(\%)$ & 38.57 & 46.57 & 45.81 \\
\hline $\mathrm{p}(\%)$ & 15 & 12.25 & 14.29 \\
\hline $\mathrm{t}(\%)$ & 26.44 & 23.54 & 21.18 \\
\hline
\end{tabular}

Source: Original

The succession that leads to the restoration of oak forests is a long-term process (Vukićević, 1965). The interpretation of these processes on Vidlič 
Mountain depends on the degree of the wildfire damage to vegetation. By monitoring the vegetation in the first three years after the wildfire on Vidlič Mountain we can distinguish two different cases:

1) oak forests that are only partially damaged by the wildfire, and

2) oak forests that are completely damaged by the wildfire.

1) At the sites of Vučje and Visočki Odorovci, where the stands of burned oak forests were studied, oak trees were not completely burned (Figure 1), and the land cover itself was affected only to a small depth. The vegetation was only partially damaged so that these stands could attain faster recovery and needed shorter recovery time to get back to the previous state, or the state before the wildfire. Physiognomy at these sites didn't change significantly compared to the state before the wildfire since oak trees in the upper layer grew green leaves immediately after the wildfire. In the second and third year, green branches appeared in the lower parts of oak trees, which means they were undergoing a gradual recovery.

Nutrients are not washed away to lower altitudes by atmospheric precipitation owing to the dense root system of woody plants. Therefore, diversity increases with the increasing altitude at these sites.

2) The situation is different in the stands in which complete destruction occurred, i.e., oak forests burned completely. Here a completely different physiognomy occurs compared to the state before the wildfire.

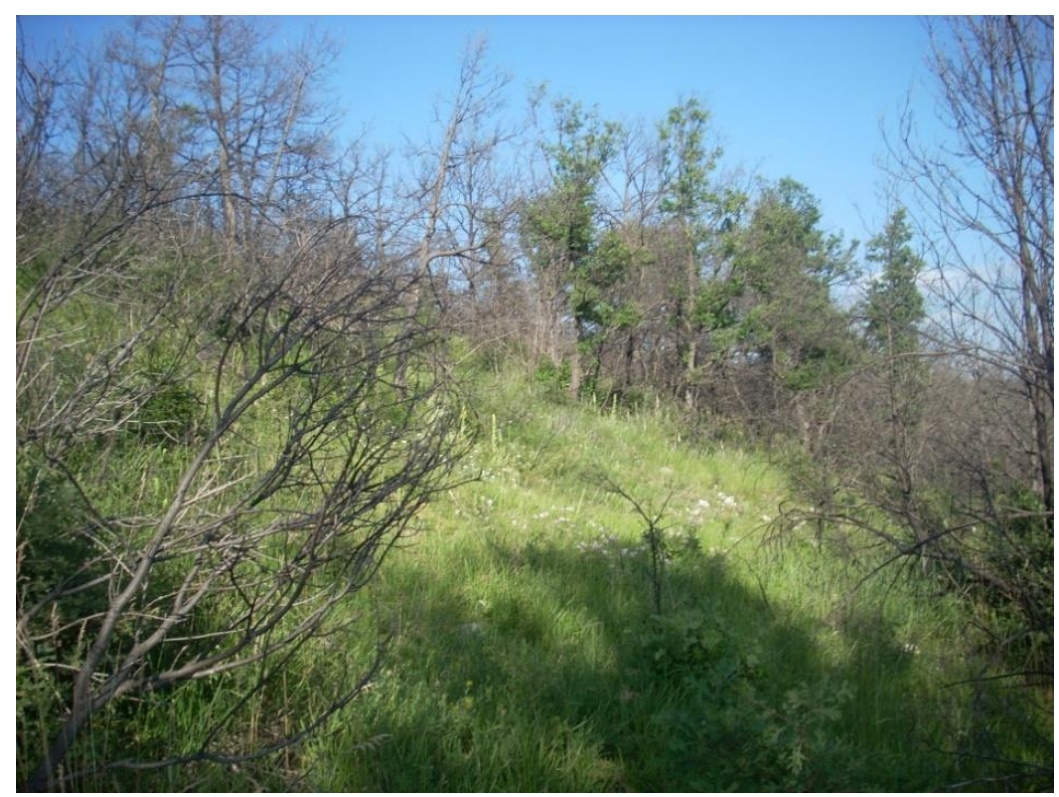

Figure 1. Partially burned oak forest at the site of Visočki Odorovci in the second year after the wildfire

Source: Original

The burned area is first inhabited by plants that take large quantities of ash as an abundant source of food for their growth and at the same time, they are 
resistant to the direct influence of sunlight and high temperatures. The groups of plants recorded in places where oak forests and Oriental hornbeam scrubs are completely burned, inhabit newly-formed open communities and belong to xerophilic and heliophilic plant species. Burned areas of oak forests now have different phenology compared to natural stands not affected by wildfires. Due to different conditions, in terms of the increased amount of light and heat, the plants of the burned areas bear flowers and fruit earlier than usual. These new plants emerge at different times. Some of them emerge in the first, while others emerge in the second or in the third year after the wildfire. As one species gives way to another, the vegetation of the burned areas goes through a series of stages, which will alternate until the original forest is restored (Vukićević-Ilić and Veslaj, 1954).

The first stage of the initial succession is characterized by the presence of therophytes. The following plants have the highest degree of presence: Orlaya grandiflora, Sideritis montana, Geranium dissectum and Althaea hirsuta (Table 1). The annual Bupleurum praealtum is recorded with a high degree of presence in the first year after the wildfire (Table 1), while it is not present in the monitored stands in the second and in the third year after the wildfire. The annual invasive thermophilic species Crepis setosa and Centaurea calcitrapa occur at low altitudes at the sites with the soil rich in nutrients. They are characterized by a narrow range of distribution and their habitats are rich in nutrients in the vicinity of human settlements. Furthermore, at not so altered sites of medium altitudes, some stands are dominated by the following species: Sideritis montana (Figure 2.), Medicago minima and Galium aparine. In the second year after the wildfire, annual species disappear from the stands, being replaced by various perrenial plants, the most abundant of which are: Festuca valesiaca, Medicago sativa subsp. falcata, Agrostis capillaris and others (Marković et al., 2016).

The next phase of the initial succession is marked by the dominance of perennial plants and different grass species, particularly on large surface areas of medium and high altitudes. These two ephemeral phases are important because they are distinguished by different floral composition and thus make two different stages.

Perrenial plants of the burned areas of oak forests and Oriental hornbeam scrubs should be observed as permanent representatives. They were present at these sites before the wildfire. The wildfire destroyed only the aboveground parts of these plants. The belowground parts not destroyed by the wildfire grew new young shoots in the first year after the fire.

In the third year after the wildfire, a large number of shoots or thin stems grew from one root of downy oak (Quercus pubescens), which are due to their small heights still classified into the shrub layer. It will take several years to build the oak forest again.

It is necessary to continue the systematic monitoring of the stands in which oak forests burned completely in order to discover when their full natural recovery can be achieved and they can return to the state before the wildfire. 


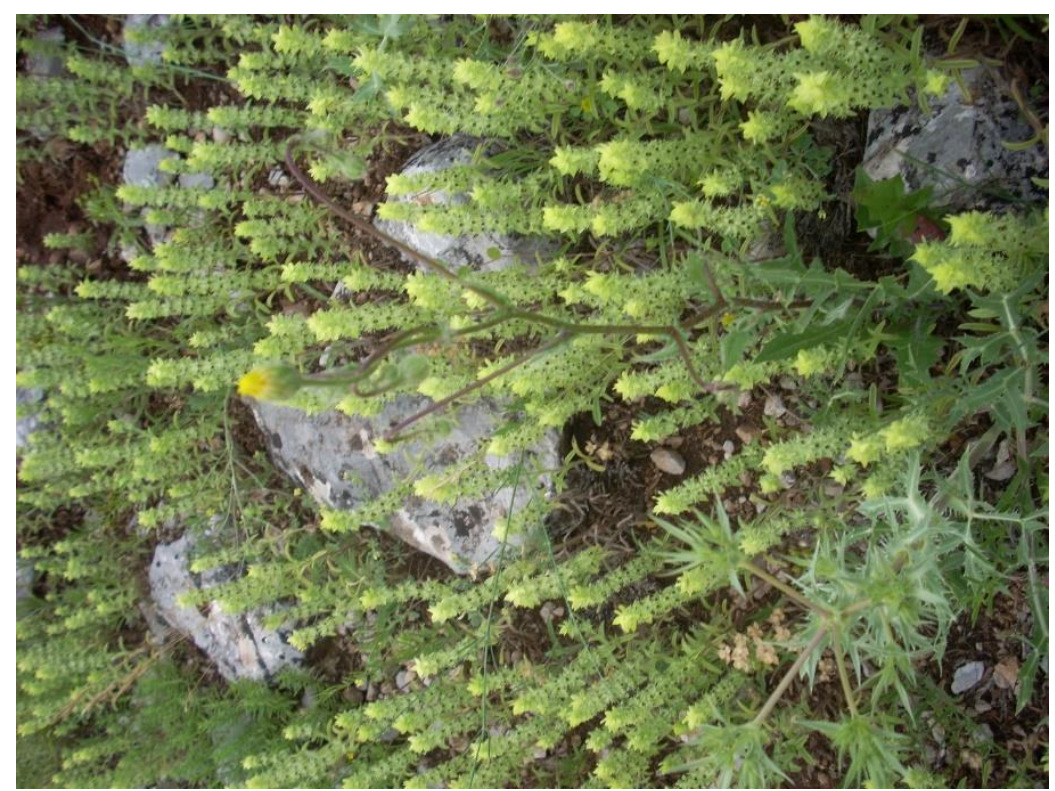

Figure 2. Dominance of the annual species of Sideritis montana at the site of Vučje in a completely burned oak forest inthe first year after the wildfire

Source: Original

Ratknić, T. et al. (2017) analyzed the profitability of the restitution of beech forests in Serbia. In addition to the tangible costs and benefits of the restitution, their research included intangible costs and benefits: its impact on the environment, biodiversity, life community and economy. They came to the conclusion that investments in the restitution of beech forests are economically acceptable. Similar research that includes economic analysis is necessary in order to provide a comprehensive approach to the possibilities of human restitution of oak forests and summarize the costs and benefits of the investments in their restitution.

\section{CONCLUSION}

After the catastrophic wildfire on Vidlič Mountain in the summer of 2007, continuous monitoring in the first three years after the wildfire revealed that the average value of the number of species and the diversity grew from year to year. At sites where vegetation was only partially damaged, a shorter time was noted to be needed to establish the original state, as it was before the wildfire. At sites where the fire swept at a higher speed and did not reach deeper in the soil, perennial plants which were present before the wildfire survived due to their well-developed belowground organs in the form of rhizomes, tubers or bulbs. In the stands in which the oak forest and Oriental hornbeam scrub burned entirely, a completely different physiognomy occurred compared to the state before the wildfire. These sites had a high share of annual plants with considerable coverage recorded in the first year after the wildfire. Their seeds were most often brought to the burned area 
by wind. In the second and third year after the wildfire, the share of perennial plants increased, especially of different grass species.

Considering the importance of the forest system, it is necessary to continue the systematic and continuous monitoring of the floristic composition and structure of these forest communities. Natural revitalization of oak forests that completely burned in the wildfire would increase their protective role, primarily in erosion control and oxygen production, and it would further recover their natural resources (medicinal and aromatic herbs, forest fruits, edible mushrooms). Revitalization of the oak forests completely burned in the wildfire is a long-lasting process. In this sense, besides the continuous monitoring of the process of restoration of these forests after the wildfire, an economic analysis would also be necessary to determine the profitability of human restitution.

\section{REFERENCES}

Braun-Blanquet, J. (1964): Pflanzensoziologie, Grundzüge der Vegetationskunde. (Berlin, itd: Spiringet: Verlag).

Диклић, Н. (1984): Животне форме биљних врста и биолошки спектар флоре СР Србије, У: Сарић М. (уредник). Вегетаиија СР Србије 1: 291-316. - Српска академија наука и уметности, Београд.

Dimitrov, T. (1990): Sistemi ocjenjivanja opasnosti od šumskih požara-pogled u budućnost, Šumarski list CXIV, 395-404.

Frančišković, S. (1963): Šuma i budućnost čovečanstva, Šumarski list 3-4: 155-160.

Glavaš, M. (2003): Šumski požari i protupožarna zaštita na području Uprave šuma Podružnice Senj od 1994-2003, Šumarski list, SUPLEMENT, 79-91.

Йорданов, Д. (Главен редактор) (1963-1995): Флора на Народна Република България I-X, Издателство на Българската Академия на Науките, София.

Јосифовић, М. (ед.) (1970-1986): Флора СР Србије І-Х, САН, Београд.

Караџић, Б., Маринковић, С. (2009): Квантитативна екологија, Фонд и Институт за биолошка истраживања.

MacGregor, D.G. (2011): The Future of Fire in Environmental Management (March, 9), Futures Journal, Forthcoming, Available at: SSRN: http:// ssrn.com/abstract=1782219

Марковић, М., Ракоњац, Љ., Пешић, Д., Николић, Б., Лучић, А. (2015): Флористичке карактеристике шибљака грабића на планини Видлич (Floristic characteristics of Oriental hornbeam scrubwood on the mountain of Vidlič), Шумарство 3: 43-56.

Marković, S., Stankov, M., Jovanović, V., Mitić, V., Stamenković, S., Ilić. M., Pešić, D. (2015): Study of oak forests and scrubs of hornbeam vegetation, metals content of Teucrium chamaedrys and soils the first year after the wildfire on Vidlič Mountain, Safety engineering Vol. 5, $\mathrm{N}^{\mathrm{o}}$ 2: 61-68. 
Марковић, М., Ракоњац, љ., Лучић, А. (2016): Храстове шуме и шибљак грабића друге године после пожара на планини Видлич (Oak forests and scrubs of hornbeam in the second year after the fire on Vidlič Mountain), Пиротски зборник 41: 57-71.

Марковић, М., Ракоњац, љ., Смиљић, М., Паповић, О. (2018): Термофилне храстове шуме и шибљак грабића треће године након пожара на планини Видлич (Thermophilous oak forests and Oriental hornbeam scrubwood in the third year after a fire on Mt. Vidlič), Шумарство 1-2: 99-110.

Министарство животне средине и просторног планирања (2008): Извештај о пожарима у заштићеним ресурсима за 2007. годину.

Pyne, S.J. (1997): Vestal fire, Seattle, WA: University of Washington Press.

Ratknić, T., Milovanović, J., Ratknić, M., Šekularac, G., Subić, J, Jeločnik, G., Poduška, Z. (2017): Analysis of the Profitability of the Restitution of Fire-Affected Beech Forests in Serbia. Appl. Ecol. Env. Res., 15 (4), 1999-2010.

Раткнић, Т. (2018): Интегрални модел заштите и управљање ризицима од шумских пожара у Републици Србији, Докторска дисертација, 1-390.

Raunkiaer, C. (1934): The life forms of plants and statistical plant geography; being the collected papers of C. Raunkiaer, translated into English by H. G. Carter, A. G. Transley and Miss Fausboll, Clarendon, London.

Šoštarić, V. (1989): Požari šuma, Šumarski list CXIII, 168-172.

The Nature Conservancy, World Wide Found for Nature and IUCN (2004): Fire, Ecosystems \& People, A preliminary Assessment of Fire as a Global Conservation Issue, October 2004.

Tutin, T.G., Heywood, W.H., Burges, N.A., Moore, D.M., Valentine, D.H., Walters, S.M., Webb, D.A. (Eds) (1964-1980) Flora Europaea, I-V. Cambrige University Press, London.

Tutin, T.G., Burges, N.A., Chater, O.A., Edmondson, J.R., Heywood, V.H., Moore, D.M., Valentine, D.H., Walters, S.M., Webb, D.A. (1993). (Eds.). Flora Europaea 1 (2nd Edition). Cambrige University Press, London.

Велковски, Н., Ацевски, Ј., Василевски, К., Симовски, Б. (2012): Ревитализација на опожарени шумски екосистеми преку природно обновување, 4 Конгрес на еколозите на Македонија со меѓународно учество, Охрид, 12-15 октобар, 2012. Книга на апстракти: 23.

Вукићевић, Е. (1965): Сукцесија вегетације и природно обнављање шума на шумским пожариштима у Србији, Гласник Шумарског факултета, Београд.

Вукићевић-Илић, Е., Веслај, В. (1954): Вегетација и земљиште на пожариштима Мајданпечке Домене, Гласник Шумарског факултета Универзитета у Београду, 8, 251-282.

Whitaker, R.H. (1972): Evolution and measurement of species diversity, Taxon 21 (2\3): 213-251. 


\title{
FLORISTIC COMPOSITION OF OAK FORESTS AND ORIENTAL HORNBEAM SCRUBS ON MI. VIDLIČ INTHE FIRST THREE YEARS AFTER A WILDFIRE
}

\author{
Ljubinko RAKONJAC, Marija MARKOVIĆ, Biljana NIKOLIĆ, \\ Aleksandar LUČIĆ, Tatjana RATKNIĆ
}

\begin{abstract}
Summary
There are few areas that completely burned on Vidlič Mountain in the wildfire in 2007 and they can be considered as newly-established sites. The largest changes in the composition of vegetation occurred in the areas where closed oak forest communities burned completely. They are located mostly on the terrain with steep slopes, so that wind can bring seeds of different plant species and thus build qualitatively new communities. They are completely different from the forests not affected by the wildfire, i.e. the forest that grew at that site before the wildfire. The presence of ash, which is derived from the burned plant parts, makes the soil deeper and increases the amount of inorganic matter in it, which is suitable for the growth and development of a large number of plants. With the time, the litterfall composed of fallen leaves and branches of woody plants that didn't burn completely during the wildfire increases the amount of organic matter in the soil produced in one season. The smaller the litterfall, the faster the increase.

During the initial stages of monitoring the recovery of the vegetation of oak forests and Oriental hornbeam scrubs, the number of species generally increases from year to year, which sometimes lasts up to the second and sometimes up to the third year after the wildfire. The species that can be found only in one releve with a large number and coverage are most important for the qualitative analysis. The annual invasive thermophilic species of Bupleurum praealtum, Centaurea calcitrapa and Sideritis montana are characteristic for the initial phase of the succession at sites where complete destruction of vegetation occurred. The coverage is the smallest in the first year after the wildfire. The interaction between the plants is small. In the second and third year after the wildfire, the coverage increases as well as the number of perennial plants and various grass species. In the oak stands which were only partially damaged, the tree layer attains a gradual increase in the vegetation height and tree diameters.

Succession, which takes many years in burnt oak forests, is in the form of a gradual alternation of communities until a closed community is built again. The time needed for the recovery depends on the duration and intensity of the wildfire, that is, the speed at which the fire pass through the area, as well as the degree of damage to the vegetation, or whether the vegetation was destroyed partially or completely. In addition, the recovery of vegetation after a wildfire depends on the proximity of the areas not affected by the wildfire, orographic factors, as well as the structure and development of soil in the areas affected by a fire.
\end{abstract}




\title{
FIORISTIČKI SASTAV HRASTOVIH ŠUMA I ŠIBLJAKA GRABIĆA PRVE TRI GODINE NAKON POŽARA NA PLANINI VIDLIČ
}

\author{
Ljubinko RAKONJAC, Marija MARKOVIĆ, Biljana NIKOLIĆ, \\ Aleksandar LUČIĆ, Tatjana RATKNIĆ
}

\begin{abstract}
Rezime
Na planini Vidlič je malo površina koje su u potpunosti izgorele u požaru 2007. godine i mogu se smatrati novim staništima. Najveće promene u sastavu vegetacije se dešavaju tamo gde su sklopljene hrastove šumske zajednice izgorele u potpunosti. Tu je teren najčešće sa većim nagibom, pa sa vetrom dođe seme različitih vrsta biljaka i dolazi do formiranja kvalitativno novih zajednica. One se u potpunosti razlikuju u odnosu na neopožarenu šumu, koja je na tom mestu bila zastupljena pre požara. Zbog prisustva pepela, koji potiče od sagorelih delova biljaka, povećava se dubina zemljišta i količina neorganske materije, a to pogoduje mnogim biljkama za rast i razvoj. Kako vreme prolazi, povećava se i količina organskih materija u sastavu zemljišta u toku jedne sezone od opalog lišća biljaka i od grana drvenastih biljaka koje nisu izgorele u potpunosti za vreme požara, od sitnih brže, a od krupnijih sporije.

Tokom inicijalnih stadijuma praćenja oporavka vegetacije hrastovih šuma i šibljaka grabića, opšta pojava je povećanje broja vrsta iz godine u godinu posle požara, koja negde ide do druge, a negde do treće godine. Vrste koje se javljaju samo u po jednom snimku sa velikom brojnošću i pokrovnošću su bitne za kvalitativnu analizu. Jednogodišnje invazivne termofilne vrste Bupleurum praealtum, Centaurea calcitrapa, Sideritis montana su karakteristične za početnu fazu inicijalnog stadijuma sukcesije na lokalitetima gde je došlo do potpunog uništenja vegetacije. Prve godine posle požara najmanja je pokrovnost. Manji je uticaj biljaka jednih na druge. Druge i treće godine dolazi do povećanja pokrovnosti kao i višegodišnjih biljaka i različitih vrsta iz porodice trava. U hrastovim sastojinama u kojima je došlo samo do delimičnog oštećenja, u spratu drveća iz godine u godinu dolazi do postupnog povećanja visine vegetacije i promera stabla.

Sukcesije, koje na požarištu hrastovih šuma traju duži niz godina, sastoje se u postepenom smenjivanju jednih zajednica drugima do ponovnog formiranja sklopljenih zajednica. Dužina oporavka zavisi od trajanja i intenziteta požara, odnosno od toga kojom je brzinom požar prešao preko podloge kao i od stepena oštećenja vegetacije, odnosno da li je vegetacija uništena delimično ili u potpunosti. Osim toga, oporavak vegetacije posle požara zavisi od blizine neopožarenim površinama, orografskih faktora, kao i od strukture i razvijenosti zemljišta u oblastima zahvaćenim požarom.
\end{abstract}

